[CONTRIBUtion From the Chemical Laboratory of Johns Hopeins University.]

\title{
THE IODOMETRIC ESTIMATION OF MERCAPTANS.
}

By J. W. Kimbali, R. L. Kramer and E. EMmEt Reid.

Received February 25, 1921.

In connection with our work on the catalytic preparation of mercaptans we needed a reliable and rapid method for estimating mercaptans. The method proposed by Klason and Carlson ${ }^{1}$ seemed to offer the best solution. They published excellent results on the aromatic mercaptans, $p$-thiocresole and $\beta$-thionaphthol as well as thioglycolic and thio-acetic acids. For the aliphatic mercaptans no results were published, but the statement is made that while the reaction is quantitative, it is difficult to show this directly because of the volatility of these compounds and the difficulty of obtaining them pure; also that the end point of the titration was sharp and permanent with good agreement when the hydriodic acid formed in the reaction was neutralized. They state that for samples of methyl, ethyl and iso-butyl mercaptans the method gave an average purity of $98 \%$, but do not give any individual determinations.

That the reaction does not go entirely to completion when the concentration of the hydrogen iodide is high is shown by a simple experiment. In $2 \mathrm{cc}$. of very pure butyl mercaptan was dissolved slowly a quantity of iodine slightly less than that required to convert it to the disulfide. There resulted a dark red iodine solution, showing the reaction to be incomplete. However, when this was shaken with $100 \mathrm{cc}$. of distilled water the red color disappeared from the disulfide layer and additional iodine had to be added to give it a permanent color. This indicated that advantage might be taken of the partition of hydrogen iodide between disulfide and water to insure completion of the reaction, eliminating the necessity for neutralizing the acid formed.

With these facts in mind, we have modified the method of Klason and Carlson somewhat and have used it in the analysis of mixtures obtained in our investigation and tested it on the purest samples of mercaptans available.

The modified method is as follows. About $35.00 \mathrm{cc}$. of $0.1 \mathrm{~N}$ iodine solution in water with potassium iodide is measured into a 250 -cc. glassstoppered bottle. A sample of about $0.25 \mathrm{~g}$. is weighed into a small weighing tube, having a well-ground glass stopper, which is loosened just before the weighing tube is dropped into the bottle containing the iodine solution. The bottle is quickly stoppered and shaken to insure release of the sample and free transfer of the iodine from the water to the mercaptan layer. The excess iodine is titrated with an $0.1 \mathrm{~N}$ thiosulfate, using starch as an indicator. The samples of methyl mercaptan were weighed in small glass bulbs in place of the weighing bottle. A

I Klason and Carlson, Ber., 39, 738 (1906). 
sharp rap against the side of the bottle was sufficient to break the bulb and release the liquid.

The table below contains the results of a series of determinations made with this method on samples of aliphatic mercaptans, and the last two columns contain, for comparison, figures by Klason and Carlson on the aromatic mercaptans. The extreme and average variations are also shown.

\begin{tabular}{|c|c|c|c|c|c|c|c|c|c|c|}
\hline No. & Methyl. & Ethyl. & Propyl, & $n$-Butyl. & $\begin{array}{c}\text { TABLE } \\
\text { Sec. } \\
\text { Butyl. }\end{array}$ & $\begin{array}{l}\text { Iso- } \\
\text { butyl. }\end{array}$ & $\begin{array}{c}\text { Iso- } \\
\text { amyl. }\end{array}$ & Phenyl. & $p$-Cresol. & $\begin{array}{c}\beta \text {-Naph- } \\
\text { thol. }\end{array}$ \\
\hline 1 & 100.03 & 98.69 & 98.79 & 99.69 & 98.33 & 98.55 & 71.27 & 99.21 & 99.89 & 100.35 \\
\hline 2 & 100.21 & 98.57 & 98.55 & 99.57 & 98.47 & 98.35 & 71.11 & 99.01 & 100.22 & 100.18 \\
\hline 3 & 100.03 & 98.73 & 98.79 & 99.56 & 98.44 & 98.48 & 71.51 & 99.19 & 99.89 & 100.00 \\
\hline 4 & 100.18 & 98.50 & 98.50 & 99.57 & 98.44 & 98.46 & 71.44 & 98.90 & 100.22 & 100.47 \\
\hline 5 & 100.07 & 98.52 & 98.82 & 99.73 & 98.55 & 98.64 & 71.33 & 98.94 & 100.01 & 99.64 \\
\hline 6 & $\ldots$ & $\cdots$ & $\cdots$ & & & & $\ldots$ & & 99.77 & 99.48 \\
\hline & .10 & 98.60 & 98.69 & 99.62 & 98.44 & 98.50 & 71.33 & 99.05 & 100.00 & 100.06 \\
\hline & & 0.2 & 0.32 & 0.17 & 0.22 & 0.29 & 0.40 & 0.31 & 0.45 & 0.99 \\
\hline & 0.01 & 0.08 & 0.13 & 0.07 & 0.05 & 0.08 & 0.11 & 0.12 & 0.15 & 0.31 \\
\hline
\end{tabular}

The fact that most of the analyses are a little low shows the difficulty of preparing mercaptans in perfectly pure condition. The methyl mercaptan may have contained traces of hydrogen sulfide, though it had been refractionated. The propyl, iso-amyl and phenyl mercaptans were imported preparations purchased a number of years ago. The iso-amyl mercaptan appears to have been purified by fractionation only. The other mercaptans had been prepared and purified with care in this laboratory at various times in the past. The butyl mercaptan had been purified with the greatest care.

The excellent agreement of the determinations among themselves establishes the method for aliphatic mercaptans and shows also that the water removes the hydriodic acid sufficiently from the disulfide layer to make the reaction quantitative.

BALTIMORE, MD.

[CONTRIBUTION FROM THE DEPARTMENT OF ChEMISTRY, UNIVERSity OF KANSAS.]

THE REACTIONS OF THE FORMAMIDINES. IX. THE SYNTHESIS OF 5-PHENYL-4-PYRAZOLE CARBOXYLIC ACID.

By F. B. DaINS AND W. S. LoNg.

Received March 3, 1921.

It has been shown in earlier papers that the amino-methylene derivatives of ethyl benzoylacetate, ethyl aceto-acetate, and acetyl acetone react with phenylhydrazine with the formation of substituted pyrazole derivatives. ${ }^{1}$

These syntheses gave a pyrazole with the carboxyl group in Position 4 and an alkyl group in Position 5 of the ring.

1 This JournaI, 31, 1155 (1909); 38, 1516 (1916); 40, 563 (1918). 